\title{
Application of Updated Sage-Husa Adaptive Kalman Filter in the Navigation of a Translational Sprinkler Irrigation Machine
}

\author{
Kenan Liu ${ }^{1, *}$, Wuyun Zhao ${ }^{1}$, Bugong Sun ${ }^{1}$, Pute Wu ${ }^{2}$, Delan Zhu ${ }^{2}$ and Peng Zhang ${ }^{1}$ \\ 1 School of Mechanical and Electrical Engineering, Gansu Agricultural University, Lanzhou 730070, China; \\ zhaowy@gsau.edu.cn (W.Z.); sunbg@gsau.edu.cn (B.S.); zhangpeng.820718@163.com (P.Z.) \\ 2 Institute of Water-Saving Agriculture in Arid Areas of China, Northwest A\&F University, Yangling 712100, \\ China; gjzwpt@vip.sina.com (P.W.); dlzhu@126.com (D.Z.) \\ * Correspondence: kenan_liu@126.com
}

Received: 16 April 2019; Accepted: 5 June 2019; Published: 17 June 2019

\begin{abstract}
Autonomous navigation for agricultural machinery has broad and promising development prospects. Kalman filter technology, which can improve positioning accuracy, is widely used in navigation systems in different fields. However, there has not been much research performed into navigation for sprinkler irrigation machines (SIMs). In this paper, firstly, a self-developed SIM is introduced. Secondly, the kinematics model is established on the platform of the self-developed SIM, and the updated Sage-Husa adaptive Kalman filter, which is an accurate and real-time self-adaptive filtering algorithm, is applied in the navigation of the SIM with the aim of improving the positioning accuracy. Finally, experiment verifications were carried out, and the results show that the self-developed SIM has good navigation performance. Besides this, the influence of abnormal observations on the positioning accuracy of the system can be restrained by using the updated Sage-Husa adaptive Kalman filter. After using the updated Sage-Husa adaptive Kalman filter for the SIM, the maximum deviation between the SIM and the predetermined path is $0.18 \mathrm{~m}$, and the average deviation is $0.08 \mathrm{~m}$; these deviations are within a reasonable range. This proves that the updated Sage-Husa adaptive Kalman filter is applicable for the navigation of sprinkler irrigation machines.
\end{abstract}

Keywords: Kalman filter; navigation; sprinkler irrigation machine; positioning accuracy

\section{Introduction}

The autonomous navigation of agricultural machinery can not only solve the problem of insufficient labor force, but also reduce energy consumption, reduce costs and improve agricultural production efficiency. However, the accuracy and reliability of agricultural machinery navigation has been restricting the level of autonomous operation of agricultural machinery. Therefore, agricultural machinery navigation technology has become one of the hot topics in current agricultural machinery research.

At present, agricultural machinery navigation methods mainly include machine vision and satellite positioning. Since machine vision is sensitive to the external environment, it is difficult to meet the requirements of autonomous navigation in different environments [1,2]. Satellite positioning navigation has become a research hotspot in recent years, but its navigation accuracy needs to be further improved $[3,4]$. Generally speaking, a single navigation mode is limited by a lack of effective information, and it is difficult to continuously provide high-quality location information. Welch et al. [5] pointed out that, due in large part to advances in digital computing, the Kalman filter has been the subject of extensive research and application, particularly in the area of autonomous or assisted navigation. Kalman filter technology, which can improve positioning accuracy, is widely used in navigation systems, and many attempts at the application of the Kalman filter in navigation have been reported. 
Regarding the application of the Kalman filter in the domain of navigation for vehicles, Gao et.al. [6] proposed a federated Kalman filter algorithm to improve the positioning accuracy of vehicle altitude. In the federated Kalman filter system, the sub-filter acts to improve the accuracy. Fan et al. [7] used adaptive Kalman filtering for vehicle laser Doppler velocimetry (LDV). It was indicated that adaptive Kalman filtering can improve the accuracy of vehicle LDV. Ko et al. [8] used an invariant extended Kalman filter for the navigation of an unmanned aerial vehicle. Tradacete et al. [9] presented a global positioning system for an autonomous electric vehicle. In this paper, two sub-systems are fused to a single system by an extended Kalman filter (EKF), reaching centimeter accuracy.

Regarding the application of the Kalman filter in the domain of object tracking, Kim et al. [10] presented a multiple-object tracking system whose design is based on multiple Kalman filters dealing with observations from two different kinds of physical sensors (radar and a charge-coupled device camera). Chen et al. [11] presented an efficient method to integrate various spatial-temporal constraints to regularize the contour tracking. In this paper, an unscented Kalman filter (UKF) is applied to estimate object parameters based on the non-linear observation model and the object dynamics. Li et al. [12] presented a cost-effective approach to track moving objects around vehicles using linearly arrayed ultrasonic sensors. Two types of tracking algorithms for the sensor array, including an extended Kalman filter (EKF) and an unscented Kalman filter (UKF), were designed for dynamic object tracking; the results showed that both EKF and UKF gave a precise tracking position. Shantaiya et al. [13] presented the tracking of multiple objects from a given video dataset. Multiple objects can be tracked simultaneously using the Kalman filter and optical flow algorithm.

Regarding the application of the Kalman filter in the domain of inertial navigation systems (INS) and global positioning systems (GPS), Narasimhappa et al. [14] modified the Sage-Husa adaptive Kalman filter to denoise the fiber optic gyroscope signal in an inertial navigation system (INS). In this work, the random error of the fiber optic gyroscope is modeled using a first order auto regressive model, and the coefficients of the model were used to initialize the transition matrix of the Sage-Husa adaptive Kalman filter. Chen et al. [15] proposed an adaptive extended Kalman filter on an INS/wireless sensor network (WSN) integration system for mobile robot indoors. Zhao et al. [16] analyzed the suitable case for the robust Kalman filter in GPS/INS systems, and the filter characteristics including parameter setting, parameter meaning, and filter convergence condition are discussed simultaneously. Liu et al. [17] proposed an information fusion method based on the adaptive Kalman filter for integrated INS/GPS navigation, and the proposed adaptive Kalman filter with an attenuation factor can restrain the measurement noise and process noise. Chen et al. [18] proposed a novel model combined with strong tracking Kalman filter and wavelet neural network algorithms for INS error compensation.

As mentioned above, the Kalman filter is widely used in different fields. However, not much research has been performed into navigation for sprinkler irrigation machines (SIMs). Besides this, Kalman filtering results usually are not optimal due to the uncertainty of the noise characteristics in the practical system [19]. The system and observation noise characteristics can reduce the estimation accuracy, and result in the reduction of the reliability and real-time performance of the filter. In order to solve these problems, the Sage-Husa adaptive filter is updated on the basis of covariance matching technology with the aim of applying it in the navigation of a translational sprinkler irrigation machine.

The rest of this paper is organized as follows. In Section 2, the self-developed SIM is introduced, and the kinematic model for the SIM is established. In Section 3, the updated Sage-Husa adaptive Kalman filter is derived and presented. In Section 4, the design of the Kalman filter for the SIM is presented. In Section 5, the application of the updated Sage-Husa adaptive Kalman filter in the navigation of the self-developed SIM is illustrated. Conclusions are drawn in Section 6.

\section{Kinematic Model of the Sprinkler Irrigation Machine}

In order to achieve accurate navigation, it is necessary to establish a precise kinematic model. In this section, the kinematic model for a self-developed translational sprinkler irrigation machine is established. 


\subsection{The Self-Developed SIM}

In this paper, the self-developed translational SIM is taken as the research platform, as shown in Figure 1.

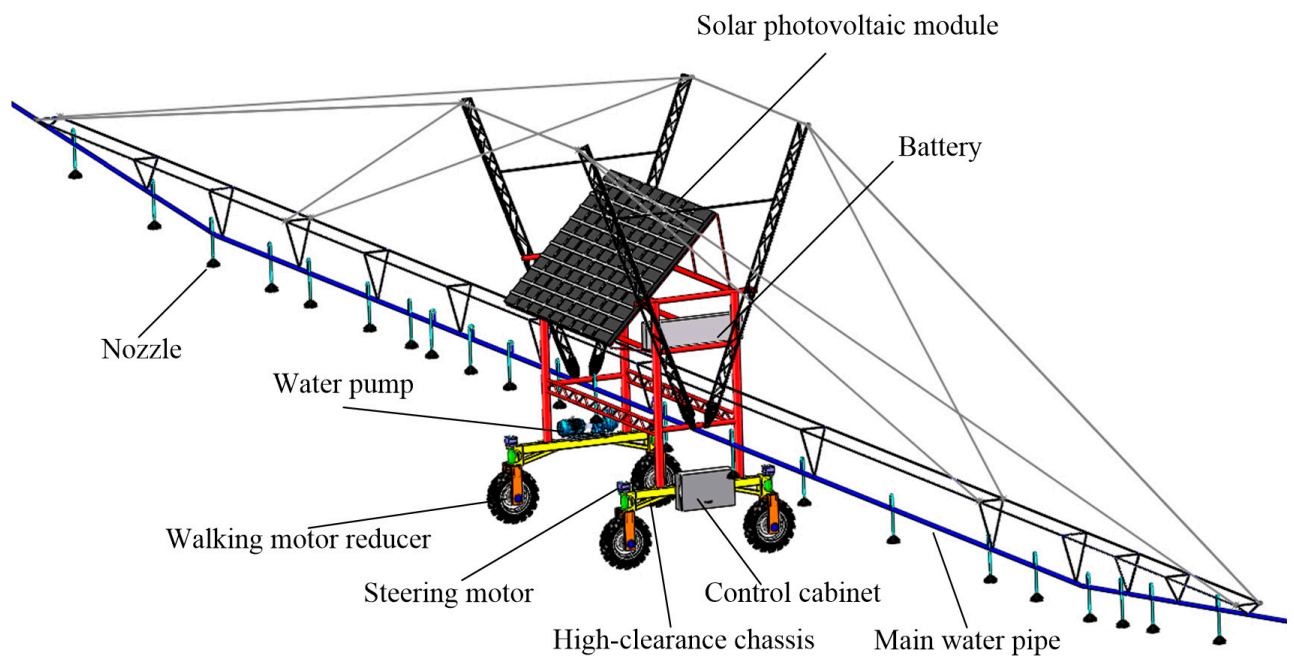

Figure 1. Structural diagrams of the self-developed translational sprinkler irrigation machine (SIM).

The self-developed SIM is composed of a solar photovoltaic power supply part, mechanical structure part and control system part. It is illustrated in Figure 1 that the self-developed SIM mainly includes a solar photovoltaic module, battery, main water pipe, water pump, steering motor, high-clearance chassis, control cabinet, walking motor reducer, nozzle and so on.

The solar photovoltaic module of the sprinkler irrigation machine converts solar radiation energy into electric energy through the photoelectric effect and stores the electric energy in its battery. Part of the stored energy is converted into mechanical energy through the stepping motor. The output torque of the motor is transferred to the power that drives the wheels to move, steered by the planetary gear reducer with a deceleration ratio i of 10 and worm gear reducer with a transmission ratio of 80:1. The other part of the electric energy is converted into the pressure energy of the pump through the horizontal centrifugal pump, which is used to draw water from the water source. The stepper motors used in the sprinkler irrigation machine are equipped with driving controllers to control the speed of the driving wheels. Considering the structure of the sprinkler, the efficiency of the driving system and the convenience of control, the wheel drive is chosen as the driving mode of the sprinkler irrigation machine. The driving mode of the wheel drive is shown in Figure 2.

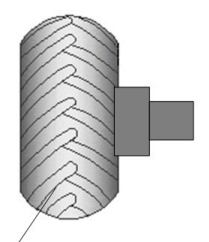

Wheel

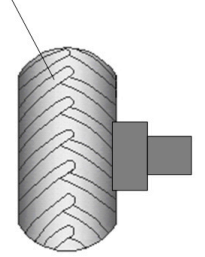

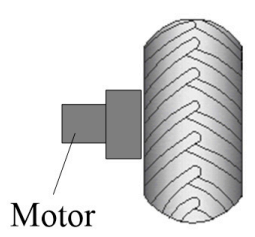

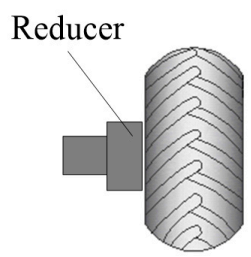

Figure 2. Driving mode of the wheel drive. 
The main technical parameters of the self-developed SIM are shown in Table 1.

Table 1. Main performance parameters of the self-developed SIM.

\begin{tabular}{cc}
\hline Parameters & Value \\
\hline Dimension (length $\times$ width $\times$ height $) / \mathrm{mm}$ & $70,000 \times 4200 \times 5000$ \\
Weight $/ \mathrm{kg}$ & 3500 \\
Spray range $/ \mathrm{m}$ & $72-76$ \\
Nozzle type & Nelson D3000 \\
Speed $/\left(\mathrm{m} / \mathrm{min}^{2}\right.$ & $\leq 1.0$ \\
Rate of flow $/\left(\mathrm{m}^{3} / \mathrm{h}\right)$ & $\leq 48$ \\
Nozzle number & 24 \\
Nozzle spacing $/ \mathrm{m}$ & 3 \\
Inlet pressure $/ \mathrm{MPa}$ & 0.1 \\
Clearance from the ground $/ \mathrm{mm}$ & 1800 \\
\hline
\end{tabular}

\subsection{Establishment of Kinematic Model}

Considering that the longitudinal speed of the SIM is small and constant during operations, and the guiding path for the navigation of the SIM is generally a straight line, the kinematic model of the self-developed SIM depicted in Figure 3 is employed for analysis.

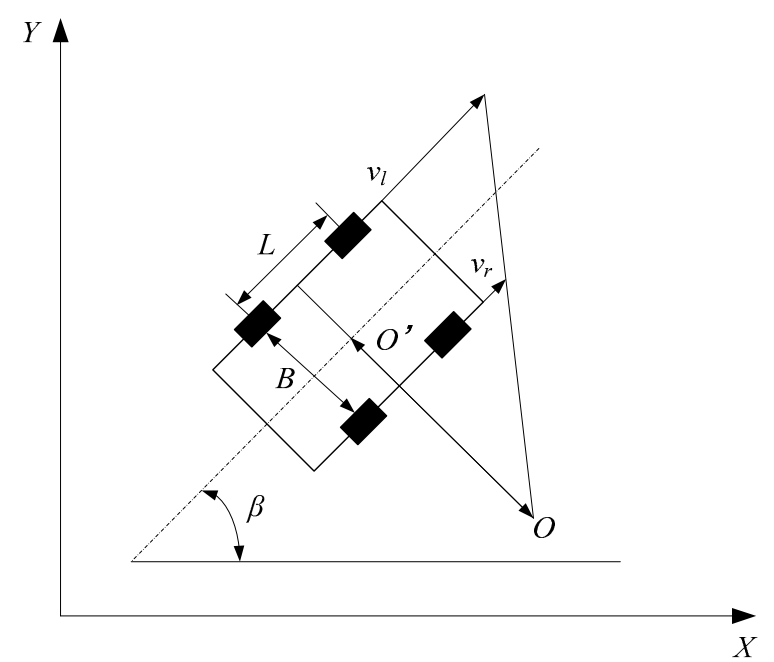

Figure 3. Kinematic model of the SIM.

As shown in Figure 3, the self-developed SIM adopts four-wheel drive. The path tracking of the SIM is realized by adjusting the wheel speed on both sides. In Figure 3, the $X-Y$ coordinate system uses the world coordinate system: $O^{\prime}$ is the center of sprinkler irrigation machine (SIM), $O$ is the instantaneous center of rotation, the distance between $O^{\prime}$ and $O$ is the turning circle of SIM, the speed at point $\mathrm{O}^{\prime}$-i.e., $v_{O}$, - can be regarded as the moving speed of the SIM, $\beta$ is the angle between the longitudinal axis of the SIM body and the $X$-axis of the world coordinate system, and $L$ is the distance between the front and rear wheels. Let the coordinates of $O^{\prime}$-i.e., $(x, y)$-be the location of the SIM. Then, the state of the sprinkler irrigation machine at any time can be expressed by the state variables $x(t), y(t)$ and $\beta(t)$.

According to Figure 3, the following equation can be obtained, expressed as

$$
\left\{\begin{array}{c}
v_{x}=v_{O}, \cos \beta \\
v_{y}=v_{O}, \sin \beta \\
\omega=\frac{v_{l}-v_{r}}{B}
\end{array}\right.
$$


where $v_{x}$ and $v_{y}$ are the velocity components along the $X$ and $Y$ direction, $v_{l}$ is the speed of the left wheel, $v_{r}$ is the speed of the right wheel, and $B$ is the distance between left wheel and right wheel.

Generally, the moving speed of the SIM is slow. In this study, it is assumed that the sprinkler irrigation machine only moves in the plane-i.e., two-dimensional motion in the $X$ and $Y$ directions-and the influence of the undulating ground and lateral slip of wheels are ignored. When the SIM moves in a plane, the speed of the $\operatorname{SIM} v_{O}$, and the steering angular velocity $\omega$ can be expressed as

$$
\begin{gathered}
v_{O}=\frac{v_{l}+v_{r}}{2} \\
\omega=\frac{v_{l}-v_{r}}{B}
\end{gathered}
$$

The path tracking of the sprinkler irrigation machine is realized by adjusting the wheel speed on both sides. When the SIM moves in a straight line, the speeds of the left and right wheel are equal; i.e., $v_{l}=v_{r}$. When SIM corrects deviation by adjusting the wheel speed on both sides, the speeds of the left and right wheel are unequal; i.e., $v_{l} \neq v_{r}$.

According to Equations (1)-(3), the kinematic model of the SIM can be obtained and expressed as

$$
\left\{\begin{array}{c}
\dot{x}=v_{x}=v_{O}, \cos \beta \\
\dot{y}=v_{y}=v_{O}, \sin \beta \\
\dot{\beta}=\omega=\frac{v_{l}-v_{r}}{B}
\end{array}\right.
$$

\section{Updated Kalman Filter Algorithm}

\subsection{Conventional Kalman Filter}

In extensive engineering applications, the actual value of the state variables of a system usually cannot be obtained directly; they can be extracted from observations which have random noise. The Kalman filter is an effective method to obtain the actual value of the system state variables by analyzing the observations of the system. Generally, the Kalman filter is a linear minimum-variance filter, which can be used to estimate and correct the system state by using an iterative algorithm.

As for a general linear system, the state equation after discretization can be expressed as follow [20]:

$$
X_{k}=A_{k, k-1} X_{k-1}+w_{k-1}
$$

where $X_{k}$ and $X_{k-1}$ are the state vector at time $k$ and time $k-1$, respectively, $A_{k, k-1}$ is the one-step state transition matrix from time $k-1$ to time $k$, and the system noise $w_{k}$ is the Gaussian white noise with an average value of zero. The expectation values of system noise can be expressed as

$$
\begin{gathered}
\mathrm{E}\left[w_{k}\right]=0 \\
\mathrm{E}\left[w_{k} w_{l}\right]=Q_{k} \delta_{k l}
\end{gathered}
$$

where $\delta_{k l}$ is the Dirac delta function.

$$
\delta_{k l}=\left\{\begin{array}{c}
1, \text { if } k=l \\
0, \text { otherwise }
\end{array}\right.
$$

where $Q_{k}$ is the process noise covariance matrix.

The observation equation of the system can be written as

$$
Z_{k}=H X_{k}+e_{k}
$$


where $Z_{k}$ is the observation at time $k, H$ is the observation matrix, $X_{k}$ is the state vector at time $k$, and the observation noise $e_{k}$ is the Gaussian white noise with zero mean.

The expectation values of observation noise can also be expressed as

$$
\begin{gathered}
\mathrm{E}\left[e_{k}\right]=0 \\
\mathrm{E}\left[e_{k} e_{l}\right]=R_{k} \delta_{k l}
\end{gathered}
$$

where $\delta_{k l}$ is the Dirac delta function, and $R$ is the observation noise covariance.

In the Kalman filter algorithm, if the state estimation and covariance matrix at the initial time $X(0)$ and $P(0)$-namely $X_{0}$ and $P_{0}$ - are given, the state estimation and its covariance matrix at time $k$-i.e., $X(k)$ and $P(k)$, namely $X_{k}$ and $P_{k},(k=1,2, \ldots)$ —can be obtained recursively according to the observation $Z(k)$, namely $Z_{k}$, at time $k$. The implementation steps of the Kalman filter are given below:

(1) Estimate the process state:

$$
X_{k, k-1}=A_{k, k-1} X_{k-1}
$$

(2) Calculate the covariance matrix of state estimation:

$$
P_{k, k-1}=A_{k, k-1} P_{k-1} A_{k, k-1}^{T}+Q_{k-1}
$$

(3) Compute the Kalman gain:

$$
K_{k}=P_{k, k-1} H_{k}^{T}\left(H_{k} P_{k, k-1} H_{k}^{T}+R\right)^{-1}
$$

(4) Update the state estimation with observation:

$$
X_{k}=X_{k, k-1}+K_{k}\left(Z_{k}-H X_{k, k-1}\right)
$$

(5) Update the error covariance:

$$
P_{k}=\left(I-K_{k} H_{k}\right) P_{k, k-1}
$$

\subsection{Sage-Husa Adaptive Kalman Filter}

The Sage-Husa adaptive filter is one of variants of the conventional Kalman filter; that is, the Sage-Husa adaptive filter algorithm is proposed based on the conventional Kalman filter algorithm. The calculation flow for the Sage-Husa adaptive filter algorithm can be described as follows [21]:

$$
\begin{gathered}
X_{k, k-1}=A_{k, k-1} X_{k-1} \\
P_{k, k-1}=A_{k, k-1} P_{k-1} A_{k, k-1}^{T}+Q_{k, k-1} \\
K_{k}=P_{k, k-1} H_{k}^{T}\left(H_{k} P_{k, k-1} H_{k}^{T}+R_{k}\right)^{-1} \\
V_{k}=Z_{k}-H_{k} X_{k, k-1} \\
X_{k}=X_{k, k-1}+K_{k} V_{k} \\
P_{k}=\left(I-K_{k} H_{k}\right) P_{k, k-1} \\
R_{k}=\left(1-d_{k}\right) R_{k-1}+d_{k}\left(V_{k} V_{k}^{T}-H_{k} P_{k, k-1} H_{k}^{T}\right)
\end{gathered}
$$

where

$$
d_{k}=(1-b) /\left(1-b^{k}\right)
$$


$d_{k}$ is the amnestic factor and $b$ is the forgetting factor in the range of 0 and $1, P_{k}$ is the covariance matrix of state estimation, $P_{k, k-1}$ is the one-step estimation variance matrix, $K_{k}$ is the filter gain, $V_{k}$ is the remainder vector, and $R_{k}$ is the observation noise covariance.

According to Equations (17)-(23), it is demonstrated that the variance matrix of the observation noise $R_{k}$ should be calculated in the filtering process for every parameter $k$ through the Sage-Husa adaptive filter algorithm, which leads to increasing filtering complexity and an extra amount of computation. Therefore, it is difficult to guarantee the real-time performance of the Sage-Husa adaptive filter algorithm in practical application. In order to apply the Sage-Husa adaptive filter to a practical situation, its real-time performance should be updated.

\subsection{Updated Sage-Husa Adaptive Kalman Filter}

The Sage-Husa adaptive Kalman filter and conventional Kalman filter both have advantages and disadvantages. The Sage-Husa adaptive Kalman filter has higher estimation accuracy; however, it has increased filter complexity, caused by large amount of computation. On the other hand, the conventional Kalman filter has higher computational efficiency, but its estimation accuracy is low. On the basis of the covariance matching method, the Sage-Husa adaptive Kalman filter algorithm can be updated in order to reduce the amount of computation and improve the real-time performance of the algorithm.

The basic idea of the covariance matching method is as follows. The actual remainder vector $V_{k}$ is verified while filtering in order to determine whether it is compatible; that is, $V_{k}$ is verified in order to determine whether abnormal filtering occurs. When the actual remainder is incompatible under the null hypothesis $Q(k-1)$ and $R(k-1)$-i.e., $Q_{k-1}$ and $R_{k-1}$ 一then $Q(k)$ and $R(k)$-i.e., $Q_{k}$ and $R_{k}$-are estimated to replace the original assumptions $Q_{k-1}$ and $R_{k-1}$ [22].

The criterion for judging filter anomaly is

$$
V_{k}^{T} V_{k}>\gamma \operatorname{tr}\left(E\left[V_{k} V_{k}^{T}\right]\right)
$$

where $\gamma$ is the reserve coefficient, $\gamma \geq 1$; $\operatorname{tr}$ represents the trace of the matrix, and $V_{k}$ is the remainder vector.

If Equation (25) is true, this indicates that the actual error will exceed the theoretical prediction value by $\gamma$ times, and the filter is divergent. When $\gamma$ is equal to 1 , this is the strictest convergence criterion.

Assuming that $R(k)=R(k-1)$, Equation (26) can be given theoretically as follows:

$$
\mathrm{E}\left[V_{k} V_{k}^{T}\right]=H_{k} P_{k, k-1} H_{k}^{T}+R_{k}
$$

Therefore, the criterion for judging filter anomaly can be rewritten as follows:

$$
V_{k} V_{k}^{T}>H_{k} P_{k, k-1} H_{k}^{T}+R_{k}
$$

In the filtering process, Equation (27) can be used to judge the filtering state. If Equation (27) is true, which means the filtering process is abnormal, then $R_{k}$ should be estimated in order to adapt it to the current filtering; otherwise, Equation (27) is not true, which means the filtering process is normal, and $R_{k}$ does not need to be estimated [23]. That is, in the $k$-th filtering process, the remainder term $V_{k}$ is used to verify $R_{k}$. If Equation (27) is true, which means that the actual remainder is incompatible with the null hypothesis $R_{k}=R_{k-1}$, then $R_{k}$ can be calculated by Equation (23) to replace $R_{k-1}$; otherwise, if Equation (27) is not true, the calculation of Equation (23) is not required, and $R_{k}$ is equal to $R_{k-1}$. Thus, the amount of computation can be reduced, and the self-adaptive estimation of $R_{k}$ can be realized.

\section{Design of Kalman Filter for Navigation of SIM}

In order to establish the Kalman filter model, the recursive relationship among the system states should first be obtained. In this study, the recursive relationship among the system states is established based on the method of dead reckoning. 


\subsection{Calculation of Navigation Position Based on Dead Reckoning}

Dead reckoning is a commonly used positioning method for autonomous navigation. In the dead reckoning method, the recursive method is employed to accumulate the moving distance of the vehicle and the direction relative to the known points, and then the position and direction of the vehicle are calculated in real time according to a certain reference position. The schematic diagram of dead reckoning is shown in Figure 4.

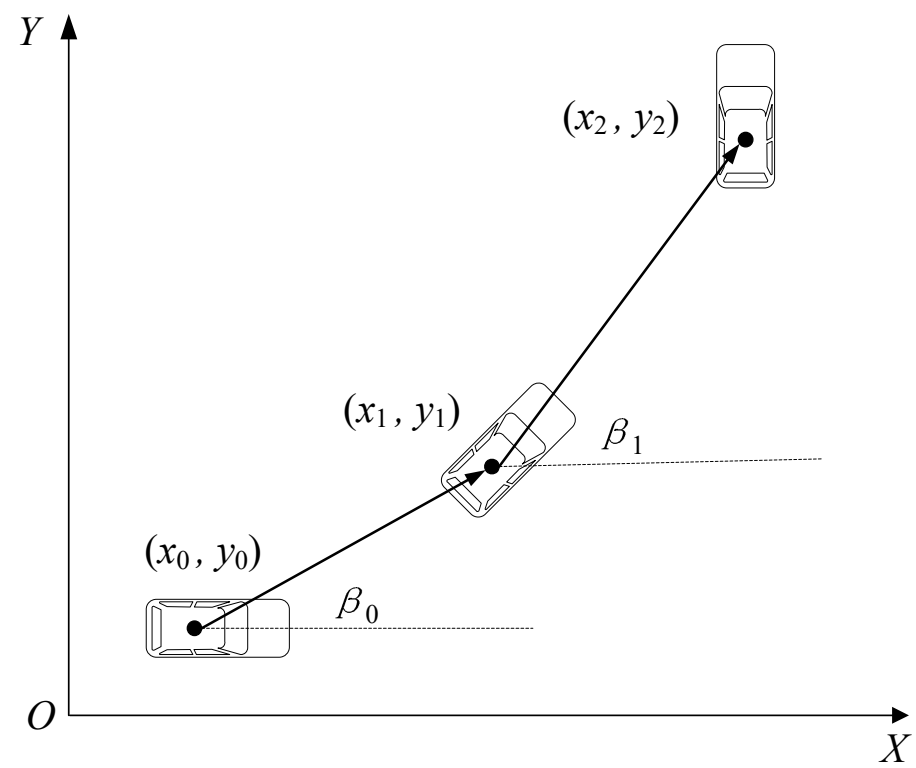

Figure 4. Schematic diagram of dead reckoning.

Assume that the heading angle $\beta$ of the sprinkler irrigation machine is unchanged during one sampling period $T$. If the initial position of the SIM is $\left(x_{0}, y_{0}\right)$, the position of the next moment of the SIM-i.e., $\left(x_{1}, y_{1}\right)$ —can be calculated by using the sampling time, the speed and heading angle of the SIM, where the speed and heading angle of the SIM are obtained by the speed sensor and electronic compass, respectively. Then, the known position $\left(x_{1}, y_{1}\right)$ can be used to calculate the position of the next moment of the SIM; i.e., $\left(x_{2}, y_{2}\right)$. The real time position during the movement of SIM can be deduced by analogy. The recurrence formula of navigation position can be written as

$$
\begin{aligned}
& x_{k}=x_{k-1}+v_{O}, T \cos \beta_{k-1} \\
& y_{k}=y_{k-1}+v_{O}, T \sin \beta_{k-1}
\end{aligned}
$$

where $x_{k}$ and $y_{k}$ are the coordinate position of the SIM at time $k, x_{k-1}$ and $y_{k-1}$ are the coordinate position of the SIM at time $k-1, v_{O}$, is the longitudinal speed of the SIM, $T$ is the sampling period, and $\beta_{k-1}$ is the heading angle of the SIM at time $k-1$.

\subsection{Design of Kalman Filter}

In this study, the Kalman filter uses the information from the speed sensor and electronic compass - i.e., the longitudinal speed $v$ and the heading angle $\beta$ of the SIM-to estimate the process state of the SIM at some time according to Equations (28) and (29). The Kalman filter uses the information from the GPS receiver and speed sensor of the SIM-i.e., the longitudinal speed $v$ and the position (values of variable $x$ and $y$ ) — to obtain feedback in the form of measurements. 


\subsubsection{Establishment of State-Transition Matrix}

In the study of navigation for the self-developed SIM, the positional variables $x, y$ and the speed variable $v$ are chosen as the system state variables; then, the state vector $X$ can be expressed as

$$
X=\left[\begin{array}{l}
x \\
y \\
v
\end{array}\right]
$$

where $x$ and $y$ are the variable values corresponding to the $X$-coordinate and $Y$-coordinate in the Gauss plane rectangular coordinate system which is converted from the WGS84 coordinate system by Gaussian projection, and $v$ is the longitudinal moving speed of the SIM; i.e., $v=v_{O}$,

According to the basic state-transition equation-i.e., Equations (5), (28), (29) and (30)-the state-transition matrix is obtained as

$$
A=\left[\begin{array}{ccc}
1 & 0 & T \cos \beta_{k-1} \\
0 & 1 & T \sin \beta_{k-1} \\
0 & 0 & 1
\end{array}\right]
$$

where $T$ is the sampling period, and $\beta_{k-1}$ is the angular components in the planar coordinates of the Gauss projection at time $k-1$.

\subsubsection{Establishment of Observation Matrix}

In the present study, the position variable $x, y$ and the speed variable $v$ are chosen as observation variables. The positional information $x$ and $y$ can be measured by the GPS receiver, and the speed information can be measured by speed sensors. Then, the observation vector $Z$ can be expressed as

$$
Z=\left[\begin{array}{l}
x \\
y \\
v
\end{array}\right]
$$

Combining the basic observation equations—i.e., Equations (9), (30) and (32)—the observation matrix can be expressed as

$$
\mathrm{H}=\left[\begin{array}{lll}
1 & 0 & 0 \\
0 & 1 & 0 \\
0 & 0 & 1
\end{array}\right]
$$

The covariance matrix of the observation noise $R_{k}$ is written as

$$
R_{k}=\left[\begin{array}{ccc}
r_{1}^{2} & 0 & 0 \\
0 & r_{2}^{2} & 0 \\
0 & 0 & r_{3}^{2}
\end{array}\right]
$$

where $r_{1}, r_{2}$ and $r_{3}$ are the standard deviation of the observation for variables $x, y$ and $v$, respectively.

\section{Application in the Navigation of the Self-Developed SIM}

In practice, the established mathematical model has difficulty reflecting the real physical process. When the mathematical model does not match the observations, the application of the Kalman filtering algorithm can lead to filter divergence. In the updated Sage-Husa adaptive Kalman filter algorithm, the self-adaptive filtering process can be realized by correcting the covariance matrix of the observation noise of the system. Therefore, the updated Sage-Husa adaptive Kalman filter algorithm has the advantages of more flexibility and reliability, and it can be used to reduce estimation error. 
In this study, the self-developed translational SIM is taken as the experimental platform. The navigation control system of the self-developed SIM is composed of a navigation controller, GPS receiver, electronic compass, speed sensor, speed controller and so on. The navigation controller is used to implement the navigation control algorithms, generate and output the control commands; the GPS receiver is employed to obtain the position information (values of $X$-coordinate and $Y$-coordinate); the electronic compass is used to measure the angular components in the planar coordinates of the Gauss projection; and the speed sensor is used to obtained the longitudinal moving speed of the SIM.

The self-developed SIM is a kind of continuous straight-line moving machine. Therefore, the experimental verification is conducted by taking linear motion as an example. In this part, the updated Sage-Husa adaptive Kalman filter algorithm is applied in the navigation.

In order to verify the navigation performance of the self-developed SIM and test the positioning accuracy of the SIM by using the updated Sage-Husa adaptive Kalman filter algorithm in navigation, the navigation tracking experiment is conducted. The experimental scenario is shown in Figure 5.

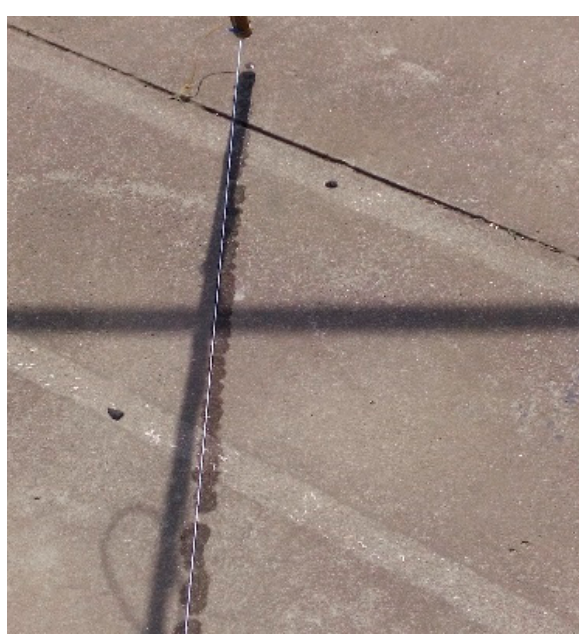

(a) Picture of predetermined path.

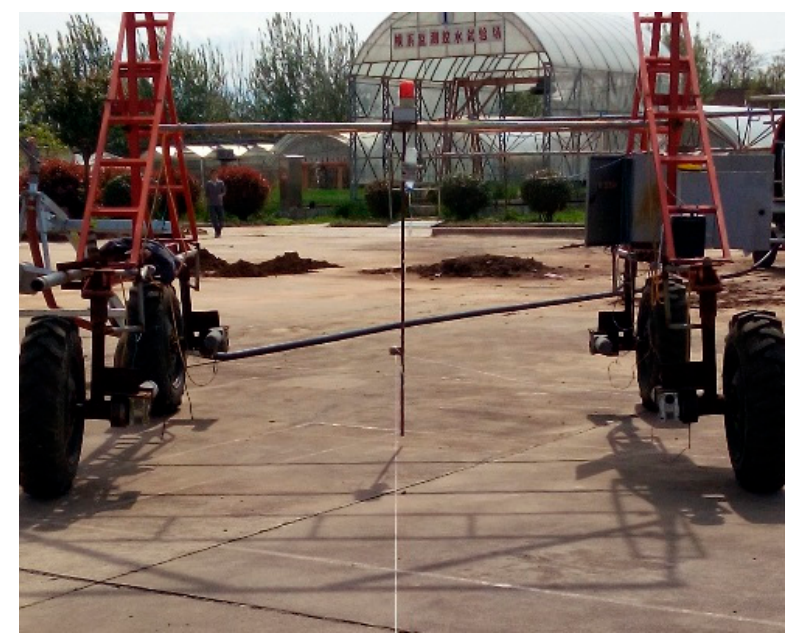

(b) Picture of navigation tracking.

Figure 5. Experimental verification scenario.

In the experiment, the SIM tracks along the predetermined path. A straight path is planned on the cement road for the verification experiment: the initial coordinates of the path are (3800970.522, 782548.0014), and the destination coordinates of the path are (3800967.693, 782516.8791). In order to facilitate the analysis and calculation, the relative coordinates, which are the difference between the collected coordinate data and initial coordinates, are used.

The initial value of the process noise covariance matrix $Q$ can be written as $Q(0)=$ $\left[\begin{array}{ccc}0.4 & & \\ & 0.4 & \\ & & 0.5\end{array}\right]$; the initial matrix for the observation noise covariance matrix $\mathrm{R}$ can be written as $R(0)=\left[\begin{array}{ccc}30 & & \\ & 30 & \\ & & 0.3\end{array}\right]$. Besides this, the initial matrix for the covariance matrix of state estimation P can be written as $\mathrm{P}(0)=\left[\begin{array}{lll}0.4 & & \\ & 0.4 & \\ & & 0.1\end{array}\right]$. The filtering results for the navigation of the SIM are shown in Figure 6. 


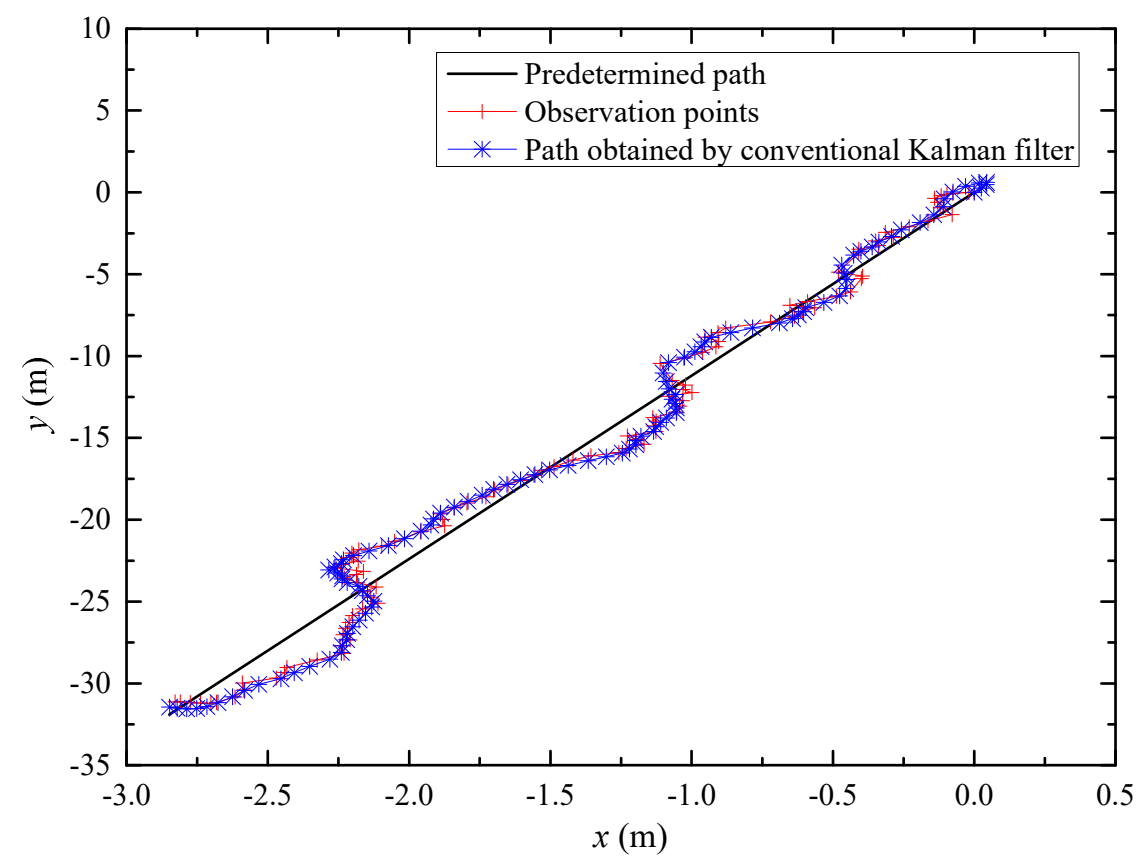

(a) Path of conventional Kalman filter

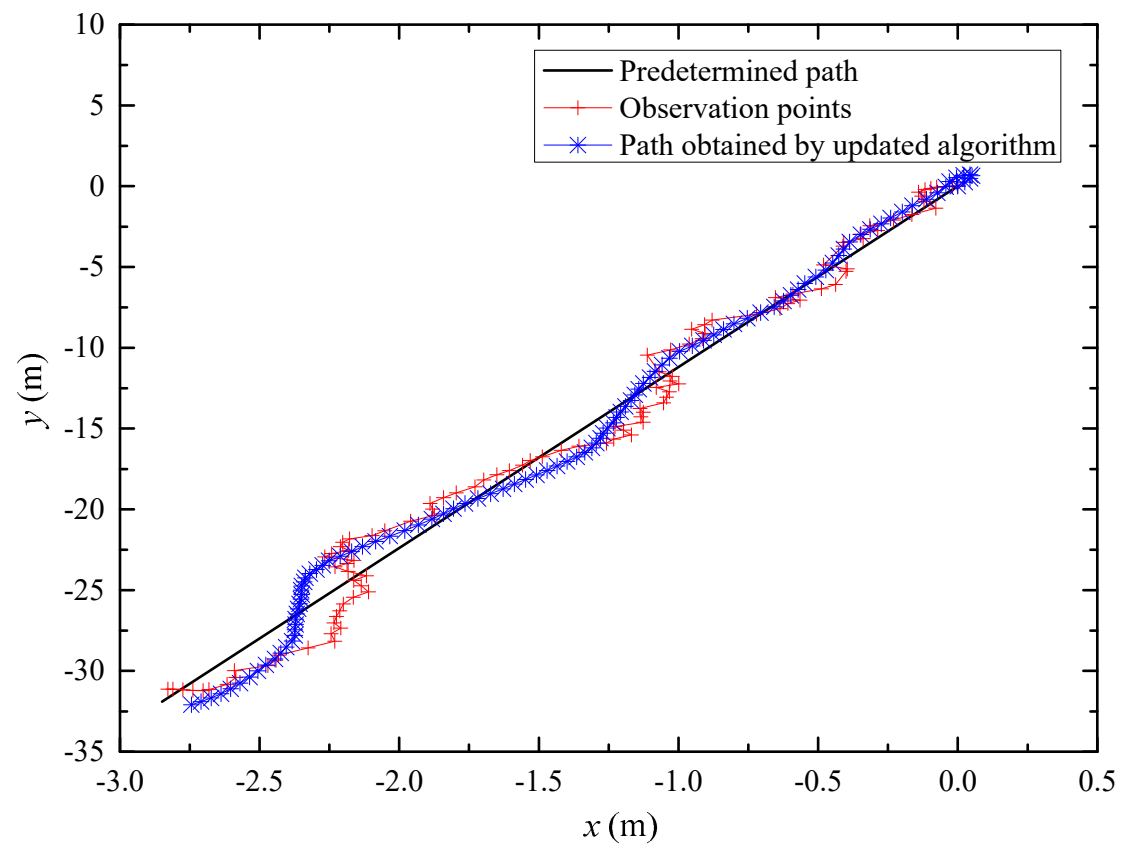

(b) Path of updated algorithm

Figure 6. Path tracking for the conventional Kalman filter and updated algorithm.

It is seen from Figure 6 that the tracking path of the updated Sage-Husa adaptive Kalman filter and conventional Kalman filter are close. This is because, during most of the path tracking time, the filtering process of the updated Sage-Husa adaptive Kalman filter is normal - that is, $R_{k}$ is adaptive to the current filtering - thus, $R_{k}$ does not need to be estimated. Therefore, there are no great differences between the filtering results of the updated Sage-Husa adaptive Kalman filter and conventional Kalman filter.

From Figure $6 a$, it can be seen that when the observations have large deviations, the data obtained by the conventional Kalman filter also have large deviations. It is seen in Figure $6 \mathrm{~b}$ that the data obtained by the updated Sage-Husa adaptive Kalman filter also have deviations; however, the deviations are 
smaller than that of the conventional Kalman filter. For a more intuitive comparison, the deviations between the predetermined path and the observation points, the path obtained by using the conventional Kalman filter (CKF) and the path obtained by using the updated algorithm (UA) are depicted in Figure 7.

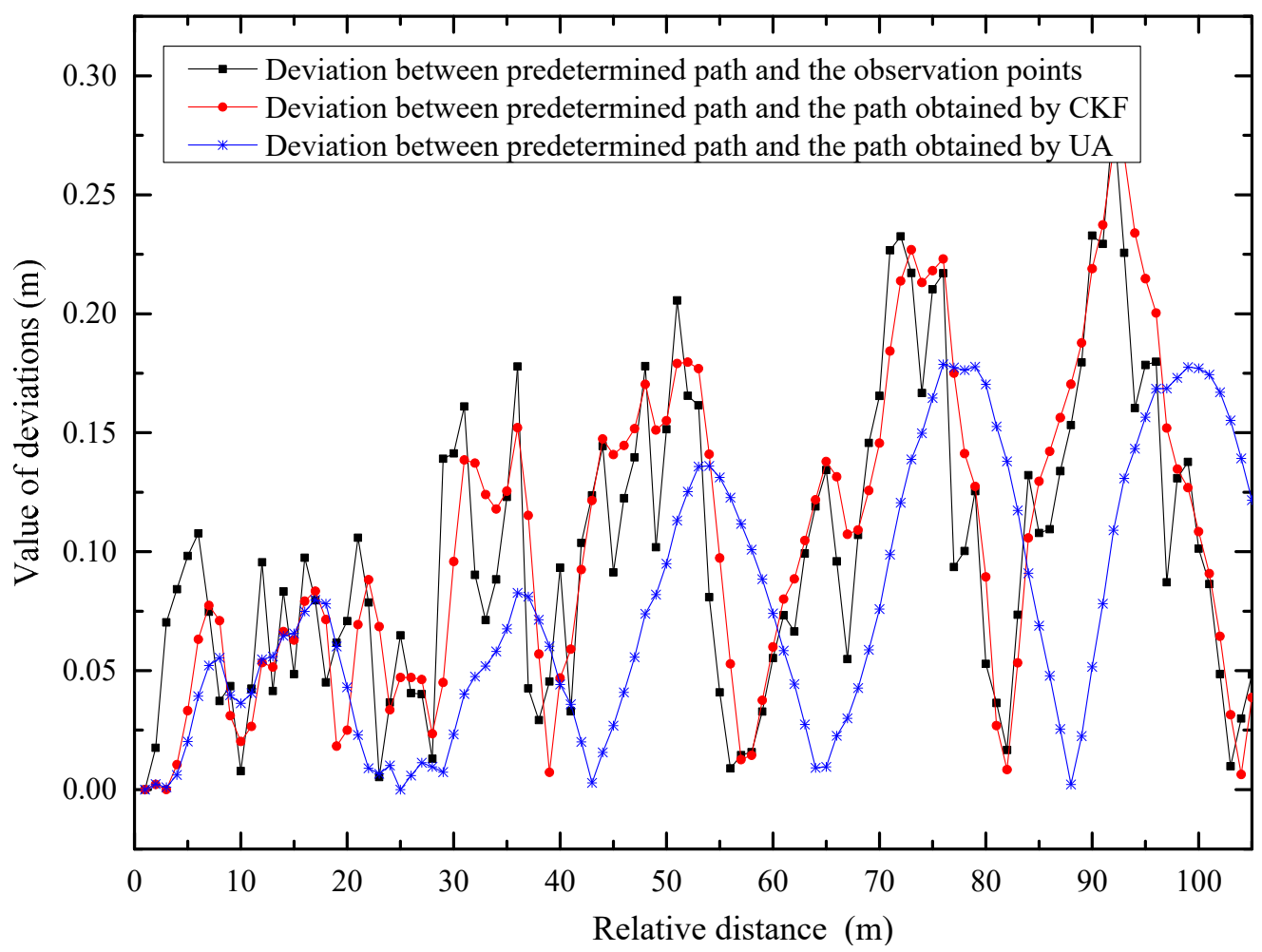

Figure 7. Deviations between the predetermined path and the observation points, the path obtained by using the conventional Kalman filter (CKF) and the updated algorithm (UA).

It is indicated in Figure 7 that the filtering precision of the updated Sage-Husa adaptive Kalman filter is higher than that of conventional Kalman filter. This is because when the filtering process is abnormal, $R_{k}$ is not adaptive to the current filtering. Thus, $R_{k}$ is estimated by using Equation (23) in order to adapt it to the current filtering process. The influence of abnormal observations on the positioning accuracy of the system can be restrained by the updated Sage-Husa adaptive Kalman filter. Accordingly, the accuracy and stability of the filter can be improved effectively.

To sum up, the self-developed SIM has good navigation performance, and the updated Sage-Husa adaptive Kalman filter can be applied in the navigation of the SIM. The average deviation, maximum deviation and deviation variance of position for the SIM, which are obtained before and after the filtering process through the updated Sage-Husa adaptive Kalman filter, are shown in Table 2.

Table 2. Error statistics before and after the filtering process.

\begin{tabular}{cccc}
\hline Items & Maximum Deviation/m & Average Deviation/m & Deviation Variance \\
\hline Before filtering & 0.28 & 0.1 & 0.004 \\
After filtering & 0.18 & 0.08 & 0.003 \\
\hline
\end{tabular}

It is indicated in Table 2 that the average deviation, maximum deviation and deviation variance become smaller after filtering through the updated Sage-Husa adaptive Kalman filter, which means the positioning accuracy of the system is improved. After using the updated Sage-Husa adaptive Kalman filter, the maximum deviation between the SIM and the predetermined path is $0.18 \mathrm{~m}$, and the average deviation is $0.08 \mathrm{~m}$; these deviations are within a reasonable range. This proves that the updated Sage-Husa adaptive Kalman filter is applicable for the navigation of sprinkler irrigation machines. 


\section{Conclusions}

The focus of this paper is to develop a translational sprinkler irrigation machine and apply the updated Sage-Husa adaptive Kalman filter to the navigation of the SIM. The following conclusions can be drawn.

1. On the platform of the self-developed translational sprinkler irrigation machine, the kinematic model for the SIM is established.

2. The updated Sage-Husa adaptive Kalman filter is applied to the navigation of the SIM. Experiment verifications were carried out, and the results show that the self-developed SIM has good navigation performance. Besides this, the influence of abnormal observations on the positioning accuracy of the system can be restrained by using the updated Sage-Husa adaptive Kalman filter.

3. The maximum deviation between the sprinkler irrigation machine and the predetermined path is $0.18 \mathrm{~m}$ and the average deviation is $0.08 \mathrm{~m}$ after using the updated filtering algorithm; the deviations are within a reasonable range. This indicates that the updated Sage-Husa adaptive Kalman filter is suitable for sprinkler irrigation machine signal processing.

Author Contributions: Data curation, K.L.; Funding acquisition, B.S.; Project administration, W.Z. and P.W.; Writing—original draft, D.Z.; Writing—review \& editing, P.Z.

Funding: This research was funded by the Special Fund for Talents of Gansu Agricultural University, grant number 2017RCZX-21 and the National Natural Science Foundation of China, grant number 61862002.

Conflicts of Interest: The authors declare no conflict of interest.

\section{References}

1. Barawid, O.C., Jr.; Mizushima, A.; Ishii, K.; Noguchi, N. Development of an autonomous navigation system using a two-dimensional laser scanner in an orchard application. Biosyst. Eng. 2007, 96, 139-149. [CrossRef]

2. Surmann, H.; Nüchter, A.; Hertzberg, J. An autonomous mobile robot with a 3D laser range finder for 3D exploration and digitalization of indoor environments. Robot. Auton. Syst. 2003, 45, 181-198. [CrossRef]

3. Nagasaka, Y.; Umeda, N.; Kanetai, Y.; Taniwaki, K.; Sasaki, Y. Autonomous guidance for rice transplanting using global positioning and gyroscopes. Comput. Electr. Agric. 2004, 43, 223-234. [CrossRef]

4. Nagasaka, Y.; Saito, H.; Tamaki, K.; Seki, M.; Kobayashi, K.; Taniwaki, K. An autonomous rice transplanter guided by global positioning system and inertial measurement unit. J. Field Robot. 2009, 26, 537-548. [CrossRef]

5. Welch, G.; Bishop, G. An Introduction to the Kalman Filter; Department of Computer Science University of North Carolina at Chapel Hill: Chapel Hill, NC, USA, 1995; pp. 41-95.

6. Gao, T.; Huang, K.; Yang, J.; Hu, Q.; Zhao, F. An Altitude Location System for Vehicle Based on Federated Kalman Filter. In Proceedings of the 5th International Conference on Systems and Informatics (ICSAI), Nanjing, China, 10-12 November 2018; IEEE: Piscataway, NJ, USA, 2018; pp. 1235-1239.

7. Fan, Z.; Sun, Q.; Du, L.; Bai, J.; Liu, J. Application of adaptive Kalman filter in vehicle laser Doppler velocimetry. Opt. Fiber Technol. 2018, 41, 163-167. [CrossRef]

8. Ko, N.; Youn, W.; Choi, I.; Song, G.; Kim, T. Features of Invariant Extended Kalman Filter Applied to Unmanned Aerial Vehicle Navigation. Sensors 2018, 18, 2855. [CrossRef] [PubMed]

9. Tradacete, M.; Sáez, Á.; Arango, J.F.; Huélamo, C.G.; Revenga, P.A.; Barea, R.; López-Guillén, E.; Bergasa, L.M. Positioning System for an Electric Autonomous Vehicle Based on the Fusion of Multi-Gnss Rtk and Odometry by Using an Extented Kalman Filter. In Proceedings of the Workshop of Physical Agents, Madrid, Spain, 22-23 November 2018; Springer: Cham, Switzerland, 2018; pp. 16-30.

10. Kim, D.Y.; Jeon, M. Data fusion of radar and image measurements for multi-object tracking via Kalman filtering. Inf. Sci. 2014, 278, 641-652. [CrossRef]

11. Chen, Y.; Huang, T.; Rui, Y. Parametric Contour Tracking Using Unscented Kalman Filter. In Proceedings of the International Conference on Image Processing, Rochester, NY, USA, 22-25 September 2002; IEEE: Piscataway, NJ, USA, 2002; Volume 3, pp. 613-616. 
12. Li, S.E.; Li, G.; Yu, J.; Liu, C.; Cheng, B.; Wang, J.; Li, K. Kalman filter-based tracking of moving objects using linear ultrasonic sensor array for road vehicles. Mech. Syst. Signal Proc. 2018, 98, 173-189. [CrossRef]

13. Shantaiya, S.; Verma, K.; Mehta, K. Multiple object tracking using Kalman filter and optical flow. Eur. J. Adv. Eng. Technol. 2015, 2, 34-39.

14. Narasimhappa, M.; Rangababu, P.; Sabat, S.L.; Nayak, J. A Modified Sage-Husa Adaptive Kalman Filter for Denoising Fiber Optic Gyroscope Signal. In Proceedings of the Annual IEEE India Conference (INDICON), Kochi, India, 7-9 December 2012; IEEE: Piscataway, NJ, USA, 2012; pp. 1266-1271.

15. Chen, X.; Xu, Y.; Li, Q. Application of adaptive extended Kalman smoothing on INS/WSN integration. Math. Probl. Eng. 2013, 2013, 130508.

16. Zhao, L.; Qiu, H.; Feng, Y. Analysis of a robust Kalman filter in loosely coupled GPS/INS navigation system. Measurement 2016, 80, 138-147. [CrossRef]

17. Liu, M.; Lai, J.; Li, Z.; Liu, J. An adaptive cubature Kalman filter algorithm for inertial and land-based navigation system. Aerosp. Sci. Technol. 2016, 51, 52-60. [CrossRef]

18. Chen, X.; Shen, C.; Zhang, W.; Tomizuka, M.; Xu, Y.; Chiu, K. Novel hybrid of strong tracking Kalman filter and wavelet neural network for GPS/INS during GPS outages. Measurement 2013, 46, 3847-3854. [CrossRef]

19. Farrell, J.; Givargis, T.; Barth, M. Differential Carrier Phase GPS-Aided INS for Automotive Applications. In Proceedings of the 1999 American Control Conference (Cat. No. 99CH36251), San Diego, CA, USA, 2-4 June 1999; IEEE: Piscataway, NJ, USA, 1999; Volume 5, pp. 3660-3664.

20. Chui, C.K.; Chen, G. Kalman Filtering; Springer International Publishing: Berlin/Heidelberg, Germany, 2017.

21. Zhao, D.; Jia, W.; Zhang, Y.; Zhao, Y.; Ji, W.; Liu, Y. Design of agricultural robot autonomous navigation control based on improved self-adaptive filter. Trans. Chin. Soc. Agric. Mach. 2015, 46, 1-6. (In Chinese)

22. Guo, L. Develop of a Low-Cost Navigation System for Autonomous Off-Road Vehicles; University of Illinois at Urbana-Champaign: Urbana-Champaign, IL, USA, 2003.

23. Lu, P.; Zhao, L.; Chen, Z. Improved Sage-Husa adaptive filtering and its application. J. Syst. Simul. 2007, 15, 3503-3505. (In Chinese)

(C) 2019 by the authors. Licensee MDPI, Basel, Switzerland. This article is an open access article distributed under the terms and conditions of the Creative Commons Attribution (CC BY) license (http://creativecommons.org/licenses/by/4.0/). 